\title{
Venous thromboembolism and lung cancer: a review
}

\author{
Carolina Vitale ${ }^{1 *}$, Maria D'Amato ${ }^{1}$, Paolo Calabrò${ }^{2}$, Anna Agnese Stanziola', Mauro Mormile ${ }^{1}$ and Antonio Molino ${ }^{1}$
}

\begin{abstract}
Venous thromboembolism (VTE) is a common complication of malignancies and epidemiological studies suggest that lung cancer belonged to the group of malignancies with the highest incidence rates of VTE. Risk factors for VTE in lung cancer patients are adenocarcinoma, NSCLC in comparison with SCLC, advanced disease, pneumonectomy, chemotherapy including antiangiogenic therapy. Other risk factors are pretreatment platelet counts and increased release of TF-positive microparticles. Elevated D-dimer levels do not necessarily indicate an increased risk of VTE but have been shown to be predictive for a worse clinical outcome in lung cancer patients. Mechanisms responsible for the increase in venous thrombosis in patients with lung cancer are not understood. Currently no biomarker is recognized as a predictor for VTE in lung cancer patients.

Although several clinical trials have reported the efficacy of antithrombotic prophylaxis in patients with lung cancer who are receiving chemotherapy, further trials are needed to assess the clinical benefit since these patients are at an increased risk of developing a thromboembolism.
\end{abstract}

Keywords: Venous thromboembolism, Lung cancer, Non small-cell lung cancer, Small-cell lung cancer, Thromboprophylaxis

\section{Introduction}

Thromboembolism is a well recognized complication of malignant disease and it is known that cancer patients have a higher incidence of venous thromboembolism (VTE), including pulmonary embolism (PE) and deep venous thrombosis (DVT), compared to the general population [1].

The incidence of thromboembolic disease in general population is relatively low, about $1-3$ per 1.000 per year [2] while among cancer patients, the occurrence of VTE is $4-7$ times higher, depending on the type and the stage of cancer [3].

It is estimated that cancer is responsible for $20 \%$ of all cases of incident VTE [4].

Epidemiological studies suggest that lung cancer belonged to the group of malignancies with the highest incidence rates [5-9] of VTE.

The association between VTE and lung cancer has been reported more than 20 years ago $[10,11]$.

\footnotetext{
* Correspondence: carolinavitale.med@gmail.com

${ }^{1}$ First Division of Pneumology, High Speciality Hospital "V. Monaldi" and University "Federico II" Medical School, Naples, Italy

Full list of author information is available at the end of the article
}

Lung cancer is the leading cause of cancer death in the United States for both men and women, with over 156,000 deaths in 2011 in the United States [9]. It is also one of the malignancies that are commonly associated with VTE, including PE, with reported incidence of VTE $3-13.8 \%$ and that of PE up to $3.8 \%[9,12,13]$.

In recent years, there has been increasing attention to the phenomenon of VTE in lung cancer patients and several studies have been made to better characterize it.

\section{Review}

Epidemiology of lung cancer-associated thrombosis It is recognized that patients with lung cancer are at risk for development of VTE. The incidence of VTE in lung cancer patients has been described in several studies.

Chew et al. [14] investgated the incidence of VTE and the risk factors associated with development of VTE in a large popuation-based study of patients with non small cell (NSCLC) and small cell lung cancer (SCLC). Among 91.933 patients with newly diagnosed lung cancer, the 1year and 2-year cumulative VTE incidence were 3.0 and $3.4 \%$, respectively, with a person-time rate of 7.2 events/ 100 patient-years during the first 6 months [14]. 
In a retrsospective analysis of a lung cancer cohort of 6732 patienst (control cohort had 17,284 patients), VTE occurred in $13,9 \%$ of the lung cancer cohort and 1,4 \% of the control cohort [15].

In another retrospective study of 1940 patients with diagnosis of lung cancer, thromboembolic events were documented in $9.8 \%$ cases, venous thromboembolic complications in $78 \%$, arterial thromboembolic complications in $27 \%$ cases. About venous thromboembolic complications, it was documented deep venous thrombosis in $55 \%$ cases and pulmonary embolism in $66 \%$ cases [16].

Recently Zhang et al. [17] described the high prevalence of VTE in patients with newly diagnosed lun cancer. VTE events occurred in $89(13,2 \%)$ of the 673 patients enrolled in the study. Forty-two (6.2 \%) patients developed lower extremity deep vein thrombosis (DVT) alone, 33 (4.9\%) patients developed pulmonary embolism (PE) and $14(2,1 \%)$ patients developed bth DVT and PE [17].

The incidence of thrombotic event was evaluated in a group of 950 patients with lung cancer by Kadlec et al. [18]. $91(8,4 \%)$ thromboembolic events were observed (34 cases of pulmonary embolism, 58 cases of deep venous thrombosis and 13 cases with simultaneous occurrence of both disease) [18]. The most common sources of venous thrombosis were low limbs $(49,74 \%)$ and superior vena cava $(5,11 \%)$; less frequent was thrombosis associated with implantable ports and venous catheters $(3,6 \%)[18]$.

In a retrospective review of lung cancer patients Alexander et al. reported that the incidence of thromboembolism was $10.8 \%$ after a medin follow up of 10 months [19].

An unclear proportion of VTE events in lung cancer patients is incidentally discovered.

White et al. [20] identified incident VTE events within 1 year before the cancer diagnosis date, by using registry data. Among 528693 cancer cases, the incidence of preceding VTE was increased over that expected in the year preceding the diagnosis of cancer, but in particular only during the 4-month period immediately preceding tha cancer diagnosis date $(p<0.001)$. Almost all of these unexpected VTE cases were significantly associated with a diagnosis of metastatic-stage cancer [20].

In a more recent study, it was observed among 207 subjects with lung cancer that one-third of VTE events was incidentally discovered [21].

Calleias et al. [22] have evaluated the prevalence of incidental VTE detected by PET-CT among cancer patients in a retrospective study. The prevalence of incidental VTE detected by PET-CT was $1.4 \%$ [22]. Patients with genitourinary malignancies, colorectal cancer and lung cancer had the highest rates of incidental VTE at PET-CT [22].

\section{Pathogenesis of VTE in cancer: evidences for lung cancer}

Mechanisms responsible for the increase in venous thrombosis in patients with cancer are not understood. Rather than one unifying mechanism, the etiology is likely multifactorial with different factors assuming lesser or greater degrees of importance depending on the clinical setting.

Over the years, researchers have investigated intrinsic properties of tumor cells that lead to a prothrombotic state. It is recognized that tumors shed membrane particles that contain procoagulant activity including tissue factor and membrane lipids that propogate the coagulation response [23, 24].

Several studies have focused on the role of Tissue Factor (TF) in the pathophysiology of cancer associated thrombosis.

Tissue factor (TF) is the physiologic initiator of coagulation and is commonly expressed in a variety of malignancies $[25,26]$. Overexpression of TF in tumor cells or elevated TF levels in association with microparticles in the systemic circulation may contribute to systemic hypercoagulability [27]. Tumor cells express TF and spontaneously release TF-positive microparticles (MPs) into the blood. MPs are small membrane vesicles that are highly procoagulant. Several studies have shown that increased levels of TF-positive MPs correlate with venous thrombosis in patients with cancer.

In the past, some studies demonstrated the expression of TF by lung cancer cells [28, 29].

Sawada et al. [30] have previously demonstrated increased TF expression in malignant cells from patients with lung cancer. Remarkably, in their study, TF expression levels correlated with the presence of metastatic tumors. Goldin-Lang et al. [31] also demonstrated increased TF expression in NSCLC, although no direct correlation between disease stage and TF expression levels was detected.

More recently the expression TF protein and/or mRNA have been documented in malignant cells or tissue in NSCLC [32].

Sato et al. [33] described a case of Trousseau's syndrome that shows the possible contribution of TF in the pathogenesis of DVT/PE in patients with lung cancer. They observed a marked increase in plasma TF level, together with positive staining of cancer cells with monoclonal anti-TF antibody. Therefore tissue factor (TF) originating from lung adenocarcinoma appeared responsible for recurrent deep vein thrombosis (DVT) and pulmonary thromboembolism (PE) [33].

Similarly, Del Conde et al. [34] reported a higher level of plasma TF in a patient with a giant-cell lung carcinoma with a severe form of Trousseau's syndrome. In this case was observed a 41-fold higher concentration of plasma TF as compared with the 
mean plasma TF concentration examined in 16 normal individuals [34].

\section{Risk factors of VTE in patients with lung cancer}

Cancer increases the risk of thromboembolic events. Risk factors, such as age, gender, bed-rest, venous catheters, surgery, chemotherapy with or without adjuvant hormone therapy, radiotherapy and infections, also increase the risk of thrombosis in cancer patients.

Rates of VTE vary substantially between cancer patients, considerably depending on clinical factors, the most important being tumor type and stage.

In this review we have focused on studies which investigated factors could be identified as predictors of vascular events in lung cancer and their prognostic significance.

\section{Haemostatic parameters}

Thrombocytosis can result in thrombosis and is frequently observed in patients with malignancies The mechanism underlying development of thrombocytosis in lung cancer patients remains unclear; one of possible mechanisms might be tumor-associated elevation of bone marrow-stimulating cytokines such as interleukin (IL)-6, IL-1 and macrophage colony stimulating factor (M-CSF). These cytokines could exhibit thrombocytosis. Incidence and prognostic significance of thrombocytosis in patients with lung cancer has been investgated in some studies [35].

Pedersen and Milman [36] examined data on platelet counts obtained in a large population (1178 patients) of patients with primary lung cancer. A high prevalence (32\%) of elevated platelet counts was observed in patients with lung cancer. The frequency of thromboembolism in patients with thrombocytosis was not statistically different from the frequency found in patients with normal platelet counts so, according the authors, thrombocytosis should not be considered as a risk factor predisposing to thromboembolism in cancer patients [36]. Thrombocytosis was associated with the tumour load according to the disease stage. Surgical debulking of the tumour resulted in significantly reduced platelet counts. Respect to survival, patients with thrombocytosis had a significantly poorer survival than patients with normal platelet counts. According to the results of this study, thrombocytosis is an indipendent prognostic factor of survival in patients with primary lung cancer [36].

Tomita et al. [35] evaluated the prognostic effect of thrombocytosis in patients with resectable NSCLC. 240 consecutive NSCLC patients, who received surgical resection, were reviewed retrospectively and the survival impact of preoperative platelet count was investigated. The 5-year survival of patients with and without thrombocytosis was 28,87 and $63,73 \%$, respectively $(p<$
0.0001). Therefore preoperative platelet count was a prognostic factor for resectable NSCLC patients [35].

These findings are supported by a more recent study [37] that assessed the prognostic value of thrombocytosis and its relation with patients undergoing surgery for lung cancer. Thrombocytosis appeared most frequently in patients with squamous cell lung cancer and among smokers. The presence of perioperative thrombocytosis in patients undergoing surgery had a negative prognostic value (the overall 5-year survival was worse in patients with thrombocytosis $(p<0,001)$ [37].

Recently Kadlec et al. [18] observed in a large cohort of lung cancer that patients with thromboembolic events showed significantly higher levels of platelet count at the time of diagnosis of lung cancer and a platelet count above $330.5 \times 10^{9}$ was associated with OR for major thromboembolic events was $3.66(2.25-5.96)$ - a statistically significant value. Thrombocytosis was found in patients with advance disease, more commonly in men, with no apparent correlation with the clinical stage of the disease [38].

On the contrary, Demirci et al. in a restrospective study of consecutive 281 patients with lung cancer did not observe a significant correlation between vascular events and thrombocytosis [39].

Plasma levels of D-dimer are elevated in cancer patients. Activation of the extrinsic coagulation system and the fibrinolytic cascade within a tumour is thought to be related with growth, invasion and metastasis. Although high D-dimer was described as a predictor of VTE in earlier works, it is now recognized that it does not necessarily indicate an increased risk of VTE in cancer patients [18].

Several studies have demonstrated that elevated Ddimer levels are associated with a poor prognosis in cancer patients. In patients with both non-small cell and small cell lung cancer, increased plasma D-dimer levels were shown to be predictive for a worse clinical outcome [40].

In addition in cancer lung patients it has been shown that D-Dimer plasma levels decrease or increase after response and progressive disease, respectively, and can act as a predictive factor of the evolution of the disease [41].

Recently Ferroni et al. [42] hypothesized that the use of a novel high-sensitive D-dimer determination could predict chemotherapy associated VTE in lung cancer patients with intermediate risk, according to Khorana's assesment model [43]. Overall 108 lung cancer patients at the start of a new platinum-based chemotherapy were evaluated. A cutoff level of 1500 was obtained by statistical analysis; it resulted in a sensitivity of $81 \%$, a specificity of $69 \%$, a positive predictive value of $31 \%$, a negative predictive value of $96 \%$ and an accuracy of $70 \%$. Patients with HSD-dimer levels above the cutoff had a worse VTE- free 
survival $(60 \%)$ compared with those with levels below the cut-off ( $95 \%, p=0.0001)$ [42]. The use of High Sensitive D-dimer determination prior to chemotherapy may help to identify cancer patients with an intermediate risk of VTE who could benefit from thromboprophylaxis [42].

With regard to other hemostatic factors, there are different results in the literature.

Gabazza et al. [11] found significantly higher levels of thrombin-antithrombin complexes III, D-dimer, and plasmin-alpha 1-antiplasmin complexes in lung cancer patients, suggesting an increased activation of coagulation and fibrinolysis.

In a prospective study Zecchina et al. [44] investigated the activation of the hemostatic factors in 45 patients undergoing chemotherapy for lung cancer. They reported no significant changes in such parameters as D-dimer, fibrinogen, and activated protein $\mathrm{C}$, however found a significant increase in platelet count 21 days after the administration of chemotherapy, correlating with the occurrence of VTE.

Elevated levels of procoagulant factors, such as lupus anticoagulant, anticardiolipin antibodies to factor VIII, and certain cytokines such as interleukin-6 and tumor necrosis factor, have also been identified as increased risk factors for VTE in patients with lung cancer $[28,29]$.

Recently Kadlec et al. in a study population of 950 lung cancer patients did not find significantly higher medians of coagulation parameters (D-dimer, fibrinogen, thrombin time and aPTT) [18].

\section{Hystological types of lung cancer}

Over the years several studies investigated the VTE risk in different hystological types of lung cancer.

In the past, autopsy and retrospective studies indicated that various adenocarcinomas are most strongly associated with VTE $[45,46]$. This has led to the widespread belief that mucin-producing adenocarcinomas are indeed the most common tumours associated with VTE. This notion was supported by the findings of several other studies [14, 15, 47]. Blom et al. [47] investigated thrombotic risk in 537 NSCLC patients and observed that the risk of VTE was 20-fold higher than in the general population (standardized morbidity ratio: 20.0 (14.6-27.4). Patients with adenocarcinoma of the lung had three-fold higher risk (incidence 66.7 per 1000 years) than patients with squamous cell carcinoma of the lung (incidence: 21.2 per 1000 years). In adeno and squamous carcinoma together, they observed 39 VTE events over 879 years of follow up for an overall incidence of VTE of 44.4 per 1000 person years [47].

In a restrospective analisys of a large cohort of patients with NSCLC and SCLC, was observed that adenocarcinoma histology ( $\mathrm{HR}=1.9$ vs. squamous cell, $95 \% \mathrm{CI}=$ 1.7-2.1) and advancing cancer stage $(\mathrm{HR}=4.0$ for metastatic vs. local disease, $95 \% \mathrm{CI}=3.4-4.6)$ were significant predictors of developing VTE within 1 years of NSCLC diagnosis [14].

Recently Kadlec et al. and Lee [18, 48] identified adenocarcinoma histology as a risk factor for development of VTE.

Tagalakis et al. [13] described a high incidence $(13,6 \%)$ of deep vein thrombisis in a cohort of 493 patients with NSCLC.

In a retrospective analysis of lung cancer patients, Alexander et al. [19] observed, that thromboembolism occurred equally in patients with non-small cell and small cell lung cancer (10.8 and $10.5 \%$ respectively), and more frequently among patients with adenocarcinoma compared to squamous cell carcinoma (14.7 and $5.3 \%$ respectively) [19].

Differently not a significant correlation between vascular events and histological type, or TNM stage was observed in a retrospective study by Demirci et al. [39].

VTE was a significant predictor of death within 2 years for both NSCLC and SCLC, HR = 2.3, $95 \% \mathrm{CI}=2.2-2.4$, and $\mathrm{HR}=1.5,95 \% \mathrm{CI}=1.3-1.7$, respectively [14].

\section{Cancer therapy}

Cancer therapy itself has been shown to increase the risk of VTE, whether it be chemotherapy, antiangiogenic therapy, or hormonal therapy.

Combined chemotherapy (in combination with radiotherapy) is the current standard treatment for advanced stage NSCLC as well as in the treatment of SCLC patients and VTE is a well known complication of anticancer therapy $[49,50]$.

How chemiotherapy contributes to VTE risk has not to our knowledge been elucidated completely but likely involves a combination of direct endothelial damage and down regolation of endogenous anticoagulants [51].

In an observational study by Blom et al. [47], the overall incidence of VTE in an ambulatory population with lung cancer starting a new chemotherapy was $1.93 \%$ over a median follow up period of 2.4 months. The rate of VTE observed in this study ( $0,8 \%$ per month) was substantially in excess of the estimated rate of approximately $0.04 \%$ per month for the entire cancer population. The data suggest that chemotherapy is associated with three-fold increased risk for VTE over and above this already elevated rate, with further increases with time on chemotherapy [47].

Khorana et al. [52] analysed data from a prospective multicentre study in 3003 patients treated with at least one cycle of chemotherapy. They reported an odds ratio for VTE of $1.86 \%$ (95 \% CI, 0.67-3.38) in lung cancer patients [52].

The results of this study are supported by Hicks et al. [53] that have conducted a pooled analysis of three clinical trials. They observed that the risk of VTE is increased in 
patients with NSCLC at all stages who receive chemotherapy, but not erlotinib or BMS-275291, whether it is given as postoperative adjuvant therapy or fort he treatment of advanced disease [53].

Numico et al. [54] prospectively assessed the occurrence of VTE in patients with NSCLC who were treated consecutively with cis-platin and gemcitabine. They observed 22 VTEs in 19 of 108 stage II-IV NSCLC patients who underwent chemotherapy (17,6 \%; 95 \% CI 10.324.8) [54].

Recently a retrospective study of lung cancer patients showed that chemotherapy-treated patients experienced thromboembolism more often than patients who did not receive chemotherapy (HR $5.795 \%$ CI 2.2-14.8) [19].

Among lung cancer patients receiving chemotherapy, it was described that the majority of VTE events occurring within 6 months of initiation of chemotherapy [55]. The presence of a VTE event is significantly associated with an increased risk of mortality [55].

With regard to antiangiogenic chemotherapy, researchers have focused on neoangiogenesis inhibition with VEGF inhibitor bevacizumab.

While some trials suggested that bevacizumab may be associated with increased risk for VTE [56], others indicate that bevacizumab is not associated with significant elevation risk in the context of contemporary chemotherapy regimens [57].

Scappaticci et al. [58] conducted a post hoc analysis of pooled data from randomized controlled trials evaluating combination treatment with bevacizuamb and chemotherapy vs chemotherapy alone in 1745 patients with colorectal, breast, or non small cell lung cancer. Compared with chemotherapy alone, bevacizumab was associated with a 2 -fold increase in arterial thromboembolic events $(p=$ 0.031 ) but was not found to be associated with an increased risk of venous thromboembolic events [58].

These data are in contrast to a recent systematic review and meta analysis by Nalluri et al. [59]; results indicated that bevacizumab was associated with an increased risk of VTE, with a relative risk (RR) of 1.33 (95\% CI, 1.13-1.56, $p<0.001)$ compared with controls [59].

Antiangiogenic agents also contribute to thrombosis, perhaps through endothelial cell and platelet activation [27].

Postoperative use of angiogenesis drugs EGFR-TKI application were high risk factors for VTE in lung cancer patients [60].

With regard to metalloproteinase inhibitor prinomastat there are divergent data.

According to Behrendt et al. [61], the use of the metalloproteinase inhibitor prinomastat in combination with chemotherapy, but not alone, resulted in approximately doubling oft he risk of VTE in advanced NSCLC patients [61].
In contrast to these data in a more recent study the addition of another metalloproteinase inhibitor BMS275291 to chemotherapy in patients with advanced NSCLC did not increase the risk [53].

The addition of aprinocarsen, a protein kinase C- alfa antisense oligonucleotide, to standard chemotherapy in patients with advanced stage NSCLC, significantly increased thromboembolism [62].

\section{Surgery}

It is known that patients undergoing surgery for cancer have a higher risk of postoperative DVT, despite thrombosis prophylaxis [63], than those having surgery for non malignant diseases [64]. Cancer patients have at least twice the risk of postoperative DVT and are known to have a more than three-fold increased risk of fatal pulmonary embolism than non cancer patients undergoing similare procedures [65].

Among patients who undergo surgical resection due to oncological processes, VTE is considered a major cause of mortality and may serve as an important predictor of survival.

With regard to lung cancer, Ziomek et al. [66] analyzed 77 patients who underwent lung resection surgery for lung cancer. They found that 20 patients $(26 \%)$ had a thromboembolic episode in the postoperative period (15 cases of DVT and 5 cases of PE) [66].

Lyman et al. [67] established that the risk of having a fatal PE after surgery is three times higher than in patients undergoing the same surgery for benign causes.

Pneumonectomy has been associated with a higher risk of VTE than lobectomy for stage I and II lung cancer, due to greater activation of coagulation, which appears from the seventh postoperative day [68].

Mason et al. [69] described a higher incidence (7.4\%) of postoperative VTE after pneumonectomy for malignancies as compared with that reported in older literature, with a peak incidence within 7 days after the operation.

Yang et al. [60] explored clinical risk factors for VTE in postoperative lung cancer patients. They described that the highest incidence of VTE is within 1 month after lung cancer surgery and high risk factors for VTE include incomplete surgical resection, postoperative use of anti-angiogenesis drugs, EGFR-TKI application and an increase in preoperative D-dimer level [60].

Recently Kadlec et al. [18] reported in a study population of 950 patients with lung cancer, that patients undergoing surgery had an incidence of $17.6 \%$ of VTE (11 and $48.4 \%$ for those undergoing radiotherapy and chemotherapy, respectively).

Differently Dentali et al. [70] have observed a low incidence of VTE in lung cancer patients undergoing surgery procedures. They described, in a series of 693 
patients who underwent thoracotomy for lung cancer under an antithrombotic prophylaxis protocol, an incidence of VTE and PE of 1.7 and $1.3 \%$, respectively, with $0.6 \%$ mortality attributed to PE [70].

Gomez-Hernandez et al. [71] have found a low prevalence of VTE in a retrospective study of 6004 patients undergoing elective thoracic surgery procedures. Among surgical procedures lobectomy and pneumonectomy were the most common and lung cancer was the main diagnosis (2245 cases). The prevalence of VTE was 0,18 \% [71]. According to Gomez-Hernandez et al., this low prevalence may be attributable to the use of thromboprophylaxis protocols, which include the use of anticoagulant drugs and mechanical measures (intermittent compression systems and elastic stockings) and early ambulation [71].

A recent published review by Christensen et al. [72] analysed 19 studies with a total of 10,660 patients with primary lung cancer undergoing operations with curative intent. The mean risk of VTE was estimated at $2.0 \%$ (range, 0.2-19\%), with a mean observation period of 16 months (range, 0.1-22). The risk of VTE seems to occur predominantly within the initial postoperative period, and subsequently the risk falls [72].

With respect to mortality, it was described that pulmonary embolism is the second cause of mortality after pneumonectomy for a malignancy and such patients have the highest risk of dying from pulmonary embolism [73]. Weder et al. [74] in their series of 176 patients who underwent pneumonectomy due to lung cancer after neoadjuvant chemotherapy, described a mortality of $3 \%$; half were attributed to pulmonary thromboembolic complications [74].

Interestingly, mortality due to VTE has been reported to occur predominantly in patients with squamous cell lung carcinoma following surgery [73].

\section{VTE prophylaxis and treatment in cancer patients: evidences for lung cancer}

In cancer patients the risk of VTE is particularly high in association with surgery for cancer and chemotherapy.

Haas et al. [75] investigated the efficacy of lowmolecular-weight heparin (certoparin 3000 IU once daily for 6 months) in the prevention of venous thromboembolism among ambulatory cancer patients. In two double blind studies, they analyzed patients with metastatic breast cancer (TOPIC -1 ) or patients with III/IV stage NSCLC (TOPIC-2). A post hoc analysis showed certoparin significantly reduced VTE in stage IV lung carcinoma (3.5\% vs $10.2 \% ; P=.032)$ without increased bleeding [75].

Zhang et al. [76] have recently publicated a systematic review and meta-analysis about the efficacy and safety of adjunctive anticoagulation in patients with lung cancer without indication for anticoagulants. The analysis of
2185 patients showed that anticoagulation reduced the incidence of VTE (RR $=0.55,95 \%$ CI $0.31-0.97 ; p=0.04$ ) and thromboembolic events $(\mathrm{RR}=0.48,95 \%$ CI $0.28-$ $0.82 ; p=0.008$ ). Furthermore anticoagulation showed significant improvement in survival at 1 year (RR 1.18, $95 \%$ CI $1.06-1.32 ; p=0.004$ ) and at 2 years (RR 1.27 , $95 \%$ CI 1.04-1.56; $p=0.02$ ), especially for patients with SCLC and prolonged life expectancy [76]. Between subcutaneus heparin and vitamin $\mathrm{K}$ antagonist, heparin was associated with a lower risk of bleeding [76].

In a retrospective analysis of lung cancer patients, patients receiving chemotherapy experienced thromboembolism more often than patients who did not receive chemotherapy (HR 5.795 \% CI 2.2-14.8) [19]. Pharmacological thromboprophylaxis was not routinely or systematically prescribed for lung cancer outpatients during any treatment phase [19]. The majority (83\%) of thromboembolic events occurred in the ambulatory care setting. It was stressed that thromboprophylaxis guidelines should be developed for the ambulatory care setting [19].

Recently the neutrophil-lymphocyte ratio (NLR) was identified as a potentially useful marker for predicting clinical outcome in patients with anticoagulation for VTE. The study has demonstrated that NLR at the time of VTE diagnosis could be a useful biomarker for predicting the response and prognosis following anticoagulation in patients with lung cancer and VTE [77].

The CARMEN study is an interesting french observational study, in which the researchers evaluated the adhesion to guidelines for treatment of VTE in hospitalized patients [78].

Among cancer patients, $64 \%$ had metastatic disease. Cancer sites were gastro-intestinal (25\%), gynecologic (23\%), pulmonary (21\%), hematological (14\%), urologic (10\%), or other (8\%) [78].

It was observed that overall adhesion to guidelines was present in $59 \%$ of patients. During initial treatment, adhesion was high but dropped during the long-term mantenance. Lung and hematological malignancies were significantly associated with the highest and lowest rates of adhesion [78].

Conversely Alexander et al. [79] made a survey among clinicians regarding appropriate thromboprophylaxis for patients with lung cancer in order to identify variation in practice and/or divergence from evidence-based clinical practice guidelines. Clinicians consistently identified patients with lung cancer as having a high thromboembolic risk in both ambulatory and surgical settings, but with differences in recommendations and variation in practice [79].

\section{Conclusions}

Venous thromboembolism, including pulmonary embolism and deep venous thrombosis, is a common occurrence 
within the cancer population. Lung cancer is one of the most common cancer in western countries with reported high incidence of VTE. VTE in hospitalized lung cancer patients is associated with longer length of stay, higher inpatient mortality rates, increased cost and greater disability upon discharge compared to other inpatient lung cancer patients [80]. Risk factors for VTE in lung cancer patients are adenocarcinoma, NSCLC in comparison with SCLC, advanced disease, pneumonectomy, chemotherapy including antiangiogenic therapy. Other risk factors are pretreatment platelet counts and increased release of TFpositive microparticles. Elevated D-dimer levels do not necessarily indicate an increased risk of VTE but have been shown to be predictive for a worse clinical outcome in lung cancer patients.

Currently no biomarker is recognized as a predictor for VTE in lung cancer patients.

Although several clinical trials have reported the efficacy of antithrombotic prophylaxis in patients with lung cancer who are receiving chemotherapy, further trials are needed to assess the clinical benefit since these patients are at an increased risk of developing a thromboembolism.

\begin{abstract}
Abbreviations
Cl: Confidence interval; DVT: Deep venous thrombosis; EGFR: Epidermal growth factor receptor; HR: Hazard ratio; LMWH: Low molecular weight heparin; NLR: Neutrophil-lymphocyte ratio; NSCLC: Non-small-cell lung cancer; OR: Odds ratio; PE: Pulmonary embolism; RR: Relative risk; SCLC: Small-cell lung cancer; TF: Tissue factor; VEGF: Vascular endothelial growth factor; VTE: Venous thromboembolism.
\end{abstract}

\section{Competing interests}

The authors declare that they have no competing interests.

\section{Authors' contributions}

All authors read and approved the final manuscript.

\section{Author details}

${ }^{1}$ First Division of Pneumology, High Speciality Hospital "V. Monaldi" and University "Federico II" Medical School, Naples, Italy. ${ }^{2}$ Department of Cardiology, High Speciality Hospital "V. Monaldi", Second University of Naples, Naples, Italy.

Received: 24 June 2015 Accepted: 27 July 2015

Published online: 15 September 2015

\section{References}

1. Shinagare AB, Okajima Y, Oxnard GR, Dipiro PJ, Johnson BE, Hatabu H, et al. Unsuspected pulmonary embolism in lung cancer patients: comparison of clinical characteristics and outcome with suspected pulmonary embolism. Lung Cancer. 2012;78(2):161-6.

2. Nordstrom M, Lindblad B, Bergqvist D, Kjellström T. A prospective study of the incidence of deep-vein thrombosis within a defined urban population. J Intern Med. 1992;232(2):155-60.

3. Falanga A. The incidence and risk of venous thromboembolism associated with cancer and nonsurgical cancer treatment. Cancer Investig. 2009;27(1):105-15,

4. Lee AY. Management of thrombosis in cancer: primary prevention and secondary prophylaxis. Br J Haematol. 2005;128:291-302.

5. Levitan N, Dowlati A, Remick SC, Tahsildar HI, Sivinski LD, Beyth R, et al. Rates of initial and recurrent thromboembolic disease among patients with malignancy versus those without malignancy: risk analysis using Medicare claims data. Medicine (Baltimore). 1999;78:285-91.
6. Shinagare AB, Guo M, Hatabu H, Krajewski KM, Andriole K, Van den Abbeele $A D$, et al. Incidence of pulmonary embolism in oncologic outpatients at a tertiary cancer center. Cancer. 2011;117(16):3860-6. PubMed: 21319153.

7. Sallah S, Wan JY, Nguyen NP. Venous thrombosis in patients with solid tumors: determination of frequency and characteristics. Thromb Haemost. 2002;87:575-57.

8. Noble S, Pasi J. Epidemiologyand pathofisiology of cancer associated thrombosis. Br J Cancer. 2010;102:S2-9.

9. Jemal A, Siegel R, Xu J, Ward E. Cancer statistics 2010. CA Cancer J Clin. 2010;60(5):277-300. PubMed: 20610543.

10. Rickles FR. Thrombosis and lung cancer. Am Rev Respir Dis. 1989;140:573-5.

11. Gabazza EC, Taguchi O, Yamakami T, Machishi M, Ibata H, Suzuki S. Evaluating prethrombotic state in lung cancer using molecular markers. Chest. 1993;103(1):196-200.

12. Tesselaar M, Osanto S. Risk of venous thromboembolism in lung cancer. Curr Opi Pul Med. 2007;13:362-7.

13. Tagalakis V, Levi D, Agulnik JS, Cohen V, Kasymjanova G, Small D. High risk of deep vein thrombosis in patients with non-small cell lung cancer: a cohort study of 493 patients. J Thorac Oncol. 2007;2(8):729-34.

14. Chew HK, Davies AM, Wun T, Harvey D, Zhou H, White RH, et al. The incidence of venous thromboembolism among patients with primary lung cancer. J Thromb Haemost. 2008;6(4):601-8.

15. Connolly GC, Dalal M, Lin J, Khorana AA. Incidence and predictors of venous thromboembolism (VTE) among ambulatory patients with lung cancer. Lung Cancer. 2012;78(3):253-8.

16. Crolow C, Samulowski M, Blum T, Kollmeier J, Schönfeld N, Bittner RC, et al. Frequency of thromboembolic complications in patients with lung cancer. Pneumologie. 2013;67(8):442-7.

17. Zhang Y, Yang Y, Chen W, Guo L, Liang L, Zhai Z, et al. Prevalence and associations of venous thromboembolism in patients with newly diagnosed lung cancer. Chest. 2014. Doi:10.1378/chest.13-2379.

18. Kadlec J, Skrickova ZM. The incidence and predictors of thromboembolic events in patients with lung cancer. Sci World J. 2014;125706:9.

19. Alexander M, Kirsa S, Wolfe R. Thromboembolism in lung cancer- an area of urgent unmet need. Lung Cancer. 2014;84(3):275-80.

20. White RH, Chew HK, Zhou H, Parikh-Patel A, Harris D, Harvey D, et al. Incidence of venous thromboembolism in the year before the diagnosis of cancer in 528,693 adults. Arch Intern Med. 2005;165:1782-7.

21. Connolly GC, Menapace L, Safadjou S, Francis CW, Khorana AA. Prevalence and clinical significance of incidental and clinically suspected venous thromboembolism in lung cancer patients. Clin Lung cancer. 2013;14(6):713-8.

22. Calleias MF, Errazuriz Jl, Castillo F, Otárola C, Riquelme C, Ortega C, et al. Incidental venous thromboembolism detected by PET-CT in patients with cancer: prevalence and impact on serviva rate. Thromb Res. 2014;133(5):750-5.

23. Hron G, Kollars $M$, Weber $H$, Sagaster $V$, Quehenberger $P$, Eichinger $S$, et al. Tissue factor-positive microparticles: cellular origin and association with coagulation activation in patients with colorectal cancer. Thromb Haemost. 2007:97(1):119-23.

24. Campello E, Spiezia L, Radu CM, Bulato C, Castelli M, Gavasso S, et al. Endothelial, platelet, and tissue factor-bearing microparticles in cancer patients with and without venous thromboembolism. Thromb Res. 2011;127(5):473-7.

25. Tesselaar ME, Romijn FP, Van Der Linden IK, Prins FA, Bertina RM, Osanto S. Microparticle-associated tissue factor activity: a link between cancer and thrombosis? J Thromb Haemost. 2007;5:520-7.

26. Khorana AA, Connolly GC. Assessing risk of venous thromboembolism in the patient with cancer. J Clin Oncology. 2009;7(29):4839-47.

27. Sousou T, Khorana A. New insights into cancer-associated thrombosis. Arterioscler Thromb Vasc Biol. 2009;29(3):316-20. doi:10.1161/ ATVBAHA.108.182196.

28. Callander NS, Varki N, Rao LV. Immunohistochemical identification of tissue factor in solid tumors. Cancer. 1992;70:1194-201.

29. Ornstein DL, Zacharski LR, Memoli VA, Kisiel W, Kudryk BJ, Hunt J, et al. Coexisting macrophageassociated fibrin formation and tumor cell urokinase in squamous cell and adenocarcinoma of the lung tissues. Cancer. 1991;68:1061-7.

30. Sawada M, Miyake S, Ohdama S, Matsubara O, Masuda S, Yakumaru K, et al. Expression of tissue factor in nonsmall-cell lung cancers and its relationship to metastasis. Br J Cancer. 1999;79(3-4):472-7. 
31. Goldin-Lang P, Tran QV, Fichtner I. Tissue factor expression pattern in human non-small cell lung cancer tissues indicate increased blood thrombogenicity and tumor metastasis. Oncol Rep. 2008;20(1):123-8.

32. Minamiya $Y$, Matsuzaki I, Sageshima M, Saito H, Taguchi $K$, Nakagawa T, et al. Expression of tissue factor mRNA and invasion of blood vessels by tumor cells in non-small cell lung cancer. Surg Today. 2004;34:1-5.

33. Sato T, Tsujino I, Ikeda D, leko M, Nishimura M. Trousseau's syndrome associated with tissue factor produced by pulmonary adenocarcinoma. Thorax. 2006;61:1009-10.

34. Del Conde I, Bharwani LD, Dietzen DJ, Pendurthi U, Thiagarajan P, López JA. Microvesicle-associated tissue factor and Trousseau's syndrome. J Thromb Haemost. 2007;5(1):70-4.

35. Tomita M, Shimizu T, Hara M, Ayabe T, Onitsuka T. Prognostic impact of thrombocytosis in resectable non-small cell lung cancer. Interactive Cardiovascular Thoracic Surgery. 2008;7:613-5.

36. Pedersen LM, Milman N. Prognostic significance of thrombocytosis in patients with primary lung cancer. Eur Respiratory J. 1996;9:1826-30.

37. Maraz A, Furak J, Varga Z. Thrombocytosis has a negative prognostic value in lung cancer. Anticancer Res. 2013;33(4):1725-9.

38. Haas S. Venous thromboembolism in medical patients-the scope of the problem. Semin Thromb Hemost. 2003;29(supplement 1):17-21.

39. Demirci NI, Turay UI, Ylmaz A, Erdogan Y, Biber Ç, Yücel H. A vascular events in lung cancer. Asian Pac J Cancer Prev. 2011;12(10):2685-7.

40. Taguchi O, Gabazza E, Yasui H, Kobayashi T, Yoshida M, Kobayashi H. Prognostic significance of plasma D-dimer levels in patients with lung cancer. Thorax. 1997;52(6):563-5.

41. Altiay G, Ciftci A, Demir M. High plasma D-dimer level is associated with decreased survival in patients with lung cancer. Clin Oncol (R Coll Radiol). 2007;19(7):494-8

42. Ferroni P, Martini F, Portarena I. Novel high-sensitive D-dimer determination predicts chemotherapy-associated venous thromboembolism in intermediate risk lung cancer patients. Clin Lung Cancer. 2012;13(6):482-7. doi:10.1016/j.cllc.2012.03.005. Epub 2012 May 15.

43. Khorana AA, Kuderer NM, Culakova E, Lyman GH, Francis CW. Development and validation of a predictive model for chemotherapy-associated thrombosis. Blood. 2008;111(10):4902-7.

44. Zecchina G, Ghio P, Bosio S, Cravino M, Camaschella C, Scagliotti GV. Reactive thrombocytosis might contribute to chemotherapy-related thrombophilia in patients with lung cancer. Clinical Lung Cancer. 2007;8(4):264-7.

45. Sack Jr GH, Levin J, Bell WR. Trousseau's syndrome and other manifestations of chronic disseminated coagulopathy in patients with neoplasms: clinical, pathophysiologic, and therapeutic features. Medicine (Baltimore). 1977;56:1-37.

46. Levi D, Tagalakis V, Cohen V, Kasymjanova G, Small D. The Risk of Deep Vein Thrombosis in Lung Cancer Patients [abstract], Proceedings of the American Thoracic Society Annual Meeting, 17-22 May 2006. Atlanta: American Thoracic Society; 2006. p. Abstract 7159.

47. Blom JW, Osanto S, Rosendaal FR. The risk of a venous thrombotic event in lung cancer patients: higher risk for adenocarcinoma than squamous cell carcinoma. J Thromb Haemost. 2004;2:1760-5.

48. Lee YG, Kim I, Lee E, Bang SM, Kang CH, Kim YT, et al. Risk factors and prognostic impact of venous thromboembolism in Asian patients with non small cell lung cancer. Thromb Haemost. 2014;111(6):1112-20.

49. Paesmans M. Benefits of chemotherapy for quality of life in patients with advanced non small cell lung cancer. Curr Opin Oncol. 2002;14:389-93.

50. Crivellari G, Monfardini S, Stragliotto S, Marino D, Aversa SM. Increasing chemotherapy in small-cell lung cancer: from dose intensity and density to megadoses. Oncologist. 2007;12:79-89.

51. Heit JA. Risk factors for venous thromboembolism. Clin Chest Med. 2003:24:1-12.

52. Khorana AA, Francis CW, Culakova E, Lyman GH. Risk factors for chemotherapy- associated venous thromboembolism in a prospective observational study. Cancer. 2005;104:2822-9.

53. Hicks LK, Cheung MC, Ding K, Hasan B, Seymour L, Le Maître A, et al. Venous thromboembolism and nonsmall cell lung cancer: a pooled analysis of National Cancer Institute of Canada Clinical Trials Group. Trials Cancer. 2009;115:5516-25.

54. Numico G, Garrone O, Dongiovanni V, Silvestris N, Colantonio I, Di Costanzo $\mathrm{G}$, et al. Prospective evaluation of major vascular events in patients with nonsmall cell lung carcinoma treated with cisplatin and gemcitabine. Cancer. 2005;103:994-9.
55. Huang H, Korn JR, Mallick R, Friedman M, Nichols C, Menzin J. Incidence of venous thromboembolism among chemotherapy-treated patients with lung cancer and its association with mortality: a retrospective database study. J Thromb Thrombolysis. 2012;34(4):446-56.

56. Shah MA, Ilson D, Kelsen DP. Thromboembolic events in gastric cancer: high incidence in patients receiving irinotecan- and bevacizumab- based therapy. J Clin Oncol. 2005;23:2574-6.

57. Hurwitz H, Fehrenbacher L, Novotny W, Cartwright T, Hainsworth J, Heim W, et al. Bevacizumab plus irinotecan, fluorouracil, and leucovorin for metastatic colorectal cancer. N Engl J Med. 2004;350:2335-42.

58. Scappaticci F, Skillings J, Holden S, Gerber HP, Miller K, Kabbinavar F, et al. Arterial thromboembolic events in patients with metastatic carcinoma treated with chemiotherapy and bevacizumab. J Natl Cancer Inst. 2007;99(16):1232-9.

59. Nalluri SR, Chu D, Keresztes R, Zhu X, Wu S. Risk of venous thromboembolism with the angiogenesis inhibitor bevacizumab in cancer patients: a meta-analysis. JAMA. 2008;300:2277-85.

60. Yang Y, Zhou Z, Niu XM, Li ZM, Chen ZW, Jian H, et al. Clinical analysis of postoperative venous thromboembolism risk factors in lung cancer patients. J Surg Oncol. 2012;106(6):736-41.

61. Behrendt CE, Ruiz RB. Venous thromboembolism among patients with advanced lung cancer randomized to prinomastat or placebo, plus chemotherapy. Thromb Haemost. 2003;90:734-7.

62. Paz-Ares L, Douillard JY, Koralewski P, Manegold C, Smit EF, Reyes JM, et al. Phase III study of gemcitabine and cisplatin with or without aprinocarsen, a protein kinase C-alpha antisense oligonucleotide, in patients with advanced-stage nonsmall-cell lung cancer. J Clin Oncol. 2006;24:1428-34.

63. ENOXACAN Study Group. Efficacy and safety of enoxaparin versus unfractionated heparin for prevention of deep vein thrombosis in elective cancer surgery: a double-blind randomized multicentre trial with venographic assessment. Br J Surg. 1997;84:1099-103.

64. Clagett GP, Reisch JS. Prevention of venous thromboembolism in general surgical patients. Results of meta-analysis. Ann Surg. 1988;208(2):227-40.

65. White $\mathrm{RH}$, Zhou H, Romanos PS. Incidence of symptomatic venous thromboembolism after different elective or urgent surgical procedures. Thromb Haemost. 2003:90:446-55.

66. Ziomek S, Read RC, Tobler HG, Harrell Jr JE, Gocio JC, Fink LM, et al. Thromboembolism in patients undergoing thoracotomy. Ann Thorac Surg. 1993;56:223-6.

67. Lyman GH, Khorana AA, Falanga A, Clarke-Pearson D, Flowers C, Jahanzeb $M$, et al. American Society of Clinical Oncology guideline: recommendations for venous thromboembolism prophylaxis and treatment in patients with cancer. J Clin Oncol. 2007;25:490-505.

68. Swiniarska J, Zekanowska E, Dancewicz M, Bella M, Szczesny TJ, Kowalewsk J. J. Pneumonectomy due to lung cancer results in a more pronounced activation of coagulation system than lobectomy. Eur J Cardiothorac Surg. 2009;36:1064-8

69. Mason DP, Quader MA, Blackstone EH, Rajeswaran J, DeCamp MM, Murthy SC, et al. Thromboembolism after pneumonectomy for malignancy: an indipendent marker of poor outcome. J Thorac Cardiovasc Surg. 2006;131:711-8

70. Dentali F, Malato A, Ageno W, Imperatori A, Cajozzo M, Rotolo N, et al. Incidence of venous thromboembolism in patients undergoing thoracotomy for lung cancer. J Thorac Cardiovasc Surg. 2008;135:705-6.

71. Gómez-Hernández T, Rodríguez-Pérez M, Novoa-Valentín N, Jiménez-López $M$, Aranda-Alcaide $J$, Varela-Simó G. Prevalence of venous thromboembolism in elective thoracic surgery maría. Arch Bronconeumol. 2013:49(7):297-302.

72. Christensen TD, Vad H, Pedersen S, Hvas AM, Wotton R, Naidu B, et al. Venous thromboembolism in patients undergoing operations for lung cancer: a systematic review. Ann Thorac Surg. 2014:97(2):394-400.

73. Kalweit G, Huwer H, Volkmer I, Petzold T, Gams E. Pulmonary embolism: a frequent cause of acute fatality after lung resection. Eur J Cardiothorac Surg. 1996;10:242-6.

74. Weder W, Collaud S, Eberhardt WE, Hillinger S, Welter S, Stahel R, et al. Pneumonectomy is a valuable treatment option after neoadjuvant therapy for stage iii non-small-cell lung cancer. J Thorac Cardiovasc Surg. 2010;139:1424-30.

75. Haas SK, Freund M, Heigener D, Heilmann L, Kemkes-Matthes B, von Tempelhoff GF, et al. Low-molecular-weight heparin versus placebo for the 
prevention of venous thromboembolism in metastatic breast cancer or stage III/IV lung cancer. Clin Appl Thromb Hemost. 2012;18(2):159-65. doi:10.1177/1076029611433769

76. Zhang J, Zhang YL, Ma KX, QU JM. Efficacy and safety of adjunctive anticoagulation in patients with lung cancer without indication for anticoagulants : a systematic review and metanalysis. Thorax. 2013;68(5):442-50. doi:10.1136/thoraxjnl-2012-202592.

77. Si G, Lee A, Lee US, Choi HJ, Kang MH, Kang JH, et al. Clinical significance of neutrophil-lymphocyte ratio in venous thromboembolism patients with lung cancer. Lung Cancer. 2014;84(1):79-85.

78. Sevestre MA, Belizna C, Durant C, Bosson JL, Vedrine L, Cajinger F, et al. Compliance with recommendations of clinical practice in the management of venous thromboembolism in cancer: the CARMEN study. J Mal Vasc. 2014;39(3):161-8. doi:10.1016/j.jmv. 2014.03.001.

79. Alexander M, Kirska S, MacManaus M, Ball D, Solomon B, Burbury K. Thromboprophylaxis for lung cancer patients-multimodality assessment of clinician practices, perceptions and decision support tools. Support Care Cancer. 2014;22(7):1915-22.

80. Steuer CE, Behera M, Kim S, Patel N, Chen Z, Pillai R, et al. Predictors and outcomes of venous thromboembolism in hospitalized lung cancer patients: a Nationwide Inpatient Sample database analysis. Lung Cancer. 2015:88:80-4

\section{Submit your next manuscript to BioMed Central and take full advantage of:}

- Convenient online submission

- Thorough peer review

- No space constraints or color figure charges

- Immediate publication on acceptance

- Inclusion in PubMed, CAS, Scopus and Google Scholar

- Research which is freely available for redistribution 